\title{
LA-UR-13-20300
}

Approved for public release; distribution is unlimited.

Title:

Author(s):

\section{LANL SAVY-4000 Field Surveillance Plan}

Kelly, Elizabeth J.

Smith, Paul Herrick

Veirs, Douglas K.

Stone, Timothy A.

Prochnow, David A.

Worl, Laura A.

Weis, Eric

Blair, Michael W.

Intended for: Report

\section{. LosAlamos}

\author{
EST. 1943
}

Disclaimer:

Los Alamos National Laboratory, an affirmative action/equal opportunity employer,is operated by the Los Alamos National

Security, LLC for the National NuclearSecurity Administration of the U.S. Department of Energy under contract DE-AC52-06NA25396.

By approving this article, the publisher recognizes that the U.S. Government retains nonexclusive, royalty-free license to

publish or reproduce the published form of this contribution, or to allow others to do so, for U.S. Government purposes.

Los Alamos National Laboratory requests that the publisher identify this article as work performed under the auspices of the

U.S. Departmentof Energy. Los Alamos National Laboratory strongly supports academic freedom and a researcher's right to publish; as an institution, however, the Laboratory does not endorse the viewpoint of a publication or guarantee its technical correctness. 
E. Kelly, P. Smith, D. K. Veirs, T. Stone, D. Prochnow, L. Worl, E. Weis, M. Blair

\section{INTRODUCTION}

The Packaging Surveillance Program section of the DOE M 441.1-1, Nuclear Material Packaging Manual (DOE, 2008) requires DOE contractors to "ensure that a surveillance program is established and implemented to ensure the nuclear material storage package continues to meet its design criteria." This report defines the near-term field surveillance plan for SAVY-4000 containers as required by the Manual. The long-term surveillance plan will be based on the results of the first several years of surveillance and the results of the lifetime extension studies.

The highest-level goal of this plan is to ensure that the SAVY-4000 containers function properly throughout their design life. To achieve this high-level goal, the surveillance sampling will meet two main objectives: (1) a high level of statistical confidence of detecting aging effects to ensure that containers continue to meet design criteria and to support determination of and/or validation of the container's design life and (2) a high level of confidence of detecting potential problems that might be introduced during packaging and/or use of the containers. The SAVY-4000 container has an initial design life of five years. The design basis is described in a safety analysis report (Anderson, et. al., 2012). As part of the first objective, the SAVY -4000 Program plans to establish the technical basis for a design life of 40 years or longer through the field shelf-life surveillance program and accelerated aging studies during the initial five year usage (Blair, 2012).

Aging impacts on containers will be evaluated through a combination of a field shelf-life program and laboratory accelerated aging studies. The latter is documented separately (Blair, 2012). The field shelf-life containers will have destructive (DE) and non-destructive evaluations (NDE). In addition, some containers that are handled as part of normal programmatic operations may have NDE's. These containers are referred to as the "items-of-opportunity" sample. If the NDE indicates a problem with the O-ring or filter, these containers will have DE evaluations.

The field shelf-life surveillance program consists of first identifying the possible "worst case" materials currently packaged in non-standard containers, Hagan containers, or SAVY-4000 containers. Worst case materials are those that are determined to be most challenging to the integrity of the containment boundary over time. The parameters used to determine worst case materials are gamma dose for the O-ring, high gamma dose and corrosive salt for the container body, and high gamma dose and the potential to generate corrosive gases for the filter. A sample of the containers packaged with worst-case material is then specified using expert judgment. The materials in the Hagan or other style containers in this worst-case sample will be repackaged into SAVY-4000 containers. The resulting field shelf-life surveillance sample consists of 24 SAVY-4000 containers packaged with worst case materials. These containers are then studied over time to assess aging impacts on the containment boundary. For the O-ring 
these results are combined with laboratory accelerated aging results to develop a model for Oring aging over time. Because the Hagan and SAVY-4000 O-rings are both made of Viton, they are likely to exhibit similarities in their aging behavior. The Hagan O-rings obtained from repackaging the worst case Hagan containers will be tested according to the DE protocol outlined here.

\section{SURVEILLANCE SAMPLING PLAN}

\section{Field Shelf-Life Surveillance Plan}

The field shelf-life surveillance consists of DE and NDE of four containers packaged with worst-case materials per year (Table 2). In addition four worst-case containers will have only NDE for 5 years. At that time a decision will be made as to continued NDE's observation or DE. Surveillance will continue after the field shelf-life surveillance. The long-term surveillance plan will be based on the results of the first several years of surveillance, including the field shelf-life surveillance, items-of-opportunity, expert judgment samples and the lifetime extension studies.

SAVY-4000 Items-of-Opportunity Sample. This sample will consist of up to 12 items per year, one container selected each operational month from those moving in and out of the vault based on programmatic needs. A container will be selected for the sample based on logistical considerations. The containers in this sample will have NDE only, unless the NDE indicates a potential problem with the O-ring. In this case the container may also have DE.

SAVY-4000 Expert Judgment (EJ) Sample. The field shelf-life surveillance and items-ofopportunity samples could be augmented with containers selected using engineering judgment. This sample has not been specified at this time. The EJ sample will consist of containers believed to have the greatest potential for degradation (e.g., containers with materials that create high thermal conditions) that are not captured in the field shelf-life and items-of-opportunity samples.

\section{FIELD SHELF-LIFE SURVEILLANCE POPULATION}

\section{Identification of Worst Case Materials Packaged in Hagan Containers}

The first step in developing the field shelf-life surveillance sample was to determine the potential worst case materials packaged in Hagan or SAVY-4000 containers. This was done by first identifying those materials with the highest dose, where dose is defined as

Calculated Dose $=\mathrm{CEDE} / \mathrm{g} *$ weight of nuclear material $(\mathrm{g}) *$ constant, $\mathrm{CEDE}=$ committed effective dose equivalent $/ \mathrm{g}$ (Smith, 2007, Table 2). 
The CEDE and the weight of the nuclear material are considered to be the most important factors affecting the integrity of the O-ring over time because they are likely to have the highest beta/gamma dose.

Figure 1 compares the calculated doses between all item description code (IDC) groups (Smith, 2007). The twelve IDC groups considered to encompass the worst case conditions for the containment boundary are identified in Table 1 with blue stars. These groups were selected because they had a reasonable number of containers with the highest calculated doses and they encompassed the salt-bearing residues. Containers with $\mathrm{Pu}-238$ were not included, since although it is a powerful alpha emitter, in most cases this material does not emit significant amounts of the more penetrating and thus more problematic beta/gamma radiation.

Figure 1. Calculated dose for each Item Description Code (IDC, see Appendix D for definitions). The blue stars indicate those categories of materials that are likely to represent the LANL inventory in terms of the chemical challenge to the container. The color of the dots on the graph indicate the isotopic composition (SMT) of the nuclear material, e.g., plutonium, uranium, etc. (see Appendix E for SMT definitions)

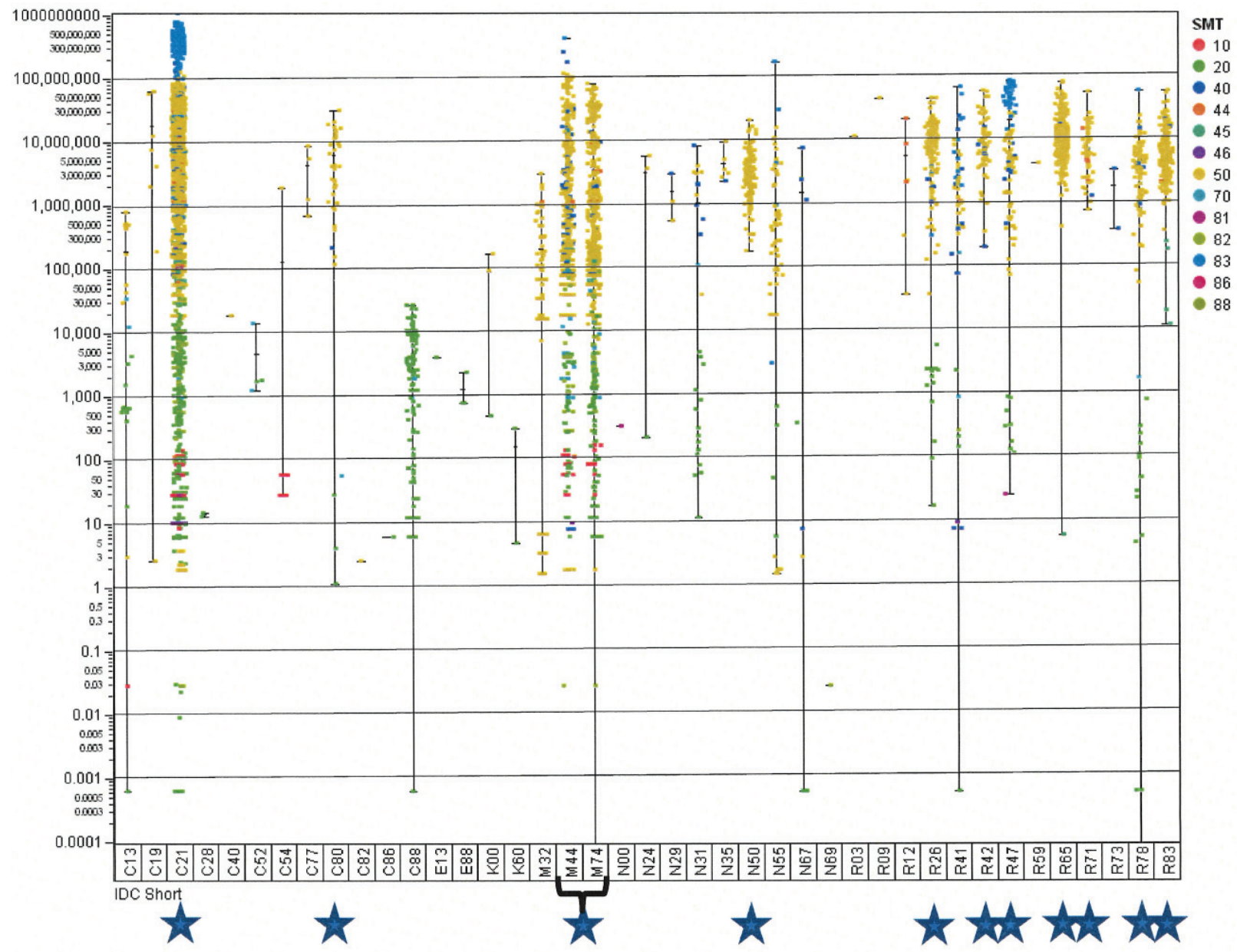


The different material characteristics, including metal, oxide and residues, are expected to bound both the corrosive (high salt impurities such as R26, R42, etc.) properties and the thermal characteristics (high americium content such as R83) of the materials in storage. The two metal IDCs (M44 and M74) were combined into one group for a total 11 groups. Once these groups were identified, experts identified two containers in each group to be evaluated over time. One additional container was selected from IDC C21 and the combined metals group for a total of 24 containers. The criteria for selecting these particular containers are a combination of the following:

- Containers with the highest beta/gamma dose listed in the remarks data field are preferred

- Small containers are preferred because the distance from the material to the O-ring and filter is small, and likely to cause more damage relative to a larger container

- Logistic considerations such as accessibility, probability that it would be repackaged into SAVY-4000 container for programmatic reasons, e.g., a Hagan with an NCR needing repackaging is preferred

- In the case of Hagan containers, older containers are preferred for maximum impact to the Hagan containment boundary

- A suitable material that is already in a SAVY container is preferred because no repackaging is necessary resulting in reduced worker exposures and lower costs.

These 24 shelf-life surveillance containers are designated as " $1{ }^{\text {st }}$ Choice" in the Pick Status column in Table 1. The " 2 nd Choice" containers may be selected if logistical, practical or programmatic considerations preclude use of the " 1 st Choice" containers for this purpose (or other comparable items if " 2 nd Choice" containers are not available).

The total of 24 containers is based on statistical considerations for detecting a trend (Appendix A) as well as cost considerations about how many DE's can be performed. The trend analysis consists of four DE's per year for five years. The remaining four containers (designated the long-term group) will have NDE evaluations each year. After three years a decision will be made about continued NDE or DE. Table 2 shows the proposed schedule for DE's and NDE's for SAVY containers derived from the containers in Table 1. Table 2 contains the IDC and Item ID for the containers given in Table 1. These containers will either be repackaged into SAVY's or are already SAVY's. The repackaging date, the surveillance date and the age of the container at the time of surveillance are also shown in Table 2. 
Table 1. Table of Shelf-Life Surveillance Containers

\begin{tabular}{|c|c|c|c|c|c|c|c|c|c|c|c|c|c|c|}
\hline IDC & $\begin{array}{c}\text { IDC } \\
\text { Description }\end{array}$ & $\begin{array}{l}\text { Project } \\
\text { Code }\end{array}$ & $\begin{array}{c}\text { Project } \\
\text { Description }\end{array}$ & Item & DMT & $\begin{array}{l}\text { Initial } \\
\text { Containe } \\
\text { r Type }\end{array}$ & Size & $\begin{array}{c}\text { SAVY } \\
\text { SN }\end{array}$ & $\begin{array}{c}\text { Containe } \\
\text { r } \\
\text { Category }\end{array}$ & $\begin{array}{l}\text { Hagan } \\
1^{\text {st }} \text { Use }\end{array}$ & $\begin{array}{c}\text { Hagan } \\
\text { Age } \\
\text { (yr) }\end{array}$ & $\begin{array}{l}\text { Calc } \\
\text { Dose }\end{array}$ & $\begin{array}{c}\text { B/G } \\
(\text { mrem/ } \\
\text { h) }\end{array}$ & $\begin{array}{c}\mathbf{B} / \mathbf{G} \\
\text { w/o } \\
\text { shield } \\
\text { (mrem/ } \\
\text { h) } \\
\end{array}$ \\
\hline \multicolumn{15}{|c|}{ First Choice Containers } \\
\hline \multirow[b]{2}{*}{$\mathrm{R} 83$} & \multirow{2}{*}{$\begin{array}{l}\text { PROCESS } \\
\text { RESIDUE, } \\
\text { MSE Salt }\end{array}$} & \multirow[b]{2}{*}{345} & \multirow{2}{*}{$\begin{array}{c}\text { MATLS } \\
\text { STAGING \& } \\
\text { DISPOSITIO } \\
\text { N- NON-NS }\end{array}$} & CABL128D & 52 & Hagan & $3 \mathrm{Qt}$ & NA & SNMC-2 & Jul-04 & 8.4 & $2 . E+07$ & NA & NA \\
\hline & & & & BLS25 & 56 & Hagan & $8 \mathrm{Qt}$ & NA & SNMC-3 & May-05 & 7.5 & 2.E+07 & 30 & 850 \\
\hline \multirow[b]{2}{*}{$\mathrm{R} 78$} & \multirow{2}{*}{$\begin{array}{c}\text { PROCESS } \\
\text { RESIDUE, } \\
\text { Sweepings/Scr } \\
\text { eenings }\end{array}$} & \multirow[b]{2}{*}{345} & \multirow{2}{*}{$\begin{array}{c}\text { MATLS } \\
\text { STAGING \& } \\
\text { DISPOSITIO } \\
\text { N- NON-NS }\end{array}$} & PO4275C1 & 52 & Hagan & $5 \mathrm{Qt}$ & NA & SNMC-1 & Aug-05 & 7.3 & $4 . \mathrm{E}+07$ & 14 & 250 \\
\hline & & & & VTB-16C1 & 52 & Hagan & $8 \mathrm{Qt}$ & NA & SNMC-2 & Aug-05 & 7.3 & 2.E+07 & 15 & NA \\
\hline \multirow[b]{2}{*}{ R71 } & \multirow[b]{2}{*}{$\begin{array}{c}\text { PROCESS } \\
\text { RESIDUE, Salt }\end{array}$} & \multirow[b]{2}{*}{345} & \multirow{2}{*}{$\begin{array}{c}\text { MATLS } \\
\text { STAGING \& } \\
\text { DISPOSITIO } \\
\text { N- NON-NS }\end{array}$} & SWPVTB15 & 52 & Hagan & $3 \mathrm{Qt}$ & NA & SNMC-2 & May-07 & 5.6 & $2 . \mathrm{E}+07$ & 70 & 70 \\
\hline & & & & PCS68B1 & 52 & Hagan & $8 \mathrm{Qt}$ & NA & SNMC-2 & Apr-08 & 4.6 & 2.E+07 & 25 & 200 \\
\hline \multirow[b]{2}{*}{ R65 } & \multirow{2}{*}{$\begin{array}{c}\text { PROCESS } \\
\text { RESIDUE, ER } \\
\text { Salt }\end{array}$} & \multirow[b]{2}{*}{345} & \multirow{2}{*}{$\begin{array}{c}\text { MATLS } \\
\text { STAGING \& } \\
\text { DISPOSITIO } \\
\text { N- NON-NS }\end{array}$} & GBS005 & 52 & Hagan & $8 \mathrm{Qt}$ & NA & SNMC-2 & Dec-04 & 7.9 & $5 . \mathrm{E}+07$ & 25 & 160 \\
\hline & & & & GBS059 & 52 & Hagan & $8 \mathrm{Qt}$ & NA & SNMC-2 & Dec-04 & 7.9 & $5 . \mathrm{E}+07$ & 17 & 400 \\
\hline \multirow[b]{2}{*}{$\mathrm{R} 47$} & \multirow{2}{*}{$\begin{array}{c}\text { PROCESS } \\
\text { RESIDUE, } \\
\text { Incinerator Ash }\end{array}$} & \multirow[b]{2}{*}{345} & \multirow{2}{*}{$\begin{array}{c}\text { MATLS } \\
\text { STAGING \& } \\
\text { DISPOSITIO } \\
\text { N- NON-NS }\end{array}$} & INCA-20 & 54 & Hagan & $8 \mathrm{Qt}$ & NA & SNMC-1 & Dec-04 & 7.9 & $3 . \mathrm{E}+07$ & 60 & 500 \\
\hline & & & & INCA-21 & 54 & Hagan & $8 \mathrm{Qt}$ & NA & SNMC-1 & Dec-04 & 8.0 & $3 . \mathrm{E}+07$ & 48 & NA \\
\hline \multirow[b]{2}{*}{$\mathrm{R} 42$} & \multirow{2}{*}{$\begin{array}{l}\text { PROCESS } \\
\text { RESIDUE, } \\
\text { DOR Salt }\end{array}$} & \multirow[b]{2}{*}{345} & \multirow{2}{*}{$\begin{array}{c}\text { MATLS } \\
\text { STAGING \& } \\
\text { DISPOSITIO } \\
\text { N- NON-NS }\end{array}$} & SLTF3123A & 52 & Hagan & $3 \mathrm{Qt}$ & NA & SNMC-1 & May-05 & 7.6 & $5 . \mathrm{E}+07$ & 10 & 200 \\
\hline & & & & BSO153 & 52 & Hagan & $5 \mathrm{Qt}$ & NA & SNMC-1 & Nov-06 & 6.0 & $3 . \mathrm{E}+07$ & 19 & NA \\
\hline \multirow{2}{*}{$\mathrm{R} 26$} & \multirow{2}{*}{$\begin{array}{l}\text { PROCESS } \\
\text { RESIDUE, } \\
\text { Filter Residue }\end{array}$} & \multirow{2}{*}{345} & \multirow{2}{*}{$\begin{array}{c}\text { MATLS } \\
\text { STAGING \& } \\
\text { DISPOSITIO } \\
\text { N- NON-NS }\end{array}$} & ROTRB9C2 & 52 & $\begin{array}{c}\text { SAVY- } \\
4000\end{array}$ & $5 \mathrm{Qt}$ & $\begin{array}{l}03110 \\
5010 \mathrm{~B} \\
\end{array}$ & $\begin{array}{c}\text { SAVY400 } \\
0 \\
\end{array}$ & Jan-00 & 0.0 & 1.E+07 & 10 & NA \\
\hline & & & & TKS1C1 & 52 & $\begin{array}{c}\text { SAVY- } \\
4000\end{array}$ & $5 \mathrm{Qt}$ & $\begin{array}{l}03110 \\
5059 \mathrm{~B} \\
\end{array}$ & $\begin{array}{c}\text { SAVY400 } \\
0 \\
\end{array}$ & Jan-00 & 0.0 & 2.E+07 & 45 & NA \\
\hline \multirow{2}{*}{ N50 } & \multirow{2}{*}{$\begin{array}{c}\text { NON- } \\
\text { COMBUSTIB } \\
\text { LE, MgO }\end{array}$} & \multirow{2}{*}{345} & \multirow{2}{*}{$\begin{array}{c}\text { MATLS } \\
\text { STAGING \& } \\
\text { DISPOSITIO } \\
\text { N- NON-NS }\end{array}$} & $\begin{array}{c}\text { ORF633956B } \\
\text { LC } \\
\end{array}$ & 52 & Hagan & $8 \mathrm{Qt}$ & NA & SNMC-3 & Dec-99 & 13.0 & $5 . \mathrm{E}+06$ & 14 & NA \\
\hline & & & & BLC9413 & 52 & Hagan & $8 \mathrm{Qt}$ & NA & SNMC-3 & Oct-99 & 13.1 & 7.E+06 & 8 & 2300 \\
\hline
\end{tabular}


E. Kelly, P. Smith, D. K. Veirs, T. Stone, D. Prochnow, L. Worl, E. Weis, M. Blair

\begin{tabular}{|c|c|c|c|c|c|c|c|c|c|c|c|c|c|c|}
\hline IDC & $\begin{array}{c}\text { IDC } \\
\text { Description }\end{array}$ & $\begin{array}{l}\text { Project } \\
\text { Code }\end{array}$ & $\begin{array}{c}\text { Project } \\
\text { Description }\end{array}$ & Item & DMT & $\begin{array}{l}\text { Initial } \\
\text { Containe } \\
\text { r Type }\end{array}$ & Size & $\begin{array}{l}\text { SAVY } \\
\text { SN }\end{array}$ & $\begin{array}{c}\text { Containe } \\
\quad \mathbf{r} \\
\text { Category }\end{array}$ & $\begin{array}{l}\text { Hagan } \\
1^{\text {st }} \text { Use }\end{array}$ & $\begin{array}{l}\text { Hagan } \\
\text { Age } \\
(\mathbf{y r})\end{array}$ & $\begin{array}{l}\text { Calc } \\
\text { Dose }\end{array}$ & $\begin{array}{c}\text { B/G } \\
(\mathbf{m r e m} / \\
\mathbf{h})\end{array}$ & $\begin{array}{c}\text { B/G } \\
\text { w/o } \\
\text { shield } \\
(\mathbf{m r e m} / \\
\text { h) }\end{array}$ \\
\hline M74 & $\begin{array}{c}\text { METAL, } \\
\text { Alloyed Metal }\end{array}$ & 362 & $\begin{array}{c}\text { PLUTONIUM } \\
\text { SUSTAINME } \\
\text { NT-LANL }\end{array}$ & 63NLBE & 52 & Hagan & $8 \mathrm{Qt}$ & NA & SNMC-3 & Aug-99 & 13.3 & 8.E+07 & NA & NA \\
\hline \multirow[b]{2}{*}{ M44 } & \multirow{2}{*}{$\begin{array}{l}\text { METAL, } \\
\text { Unalloyed } \\
\text { Metal }\end{array}$} & 311 & NEST- LANL & NAB183-2 & 52 & $\begin{array}{l}\text { SAVY- } \\
4000 \\
\end{array}$ & $3 \mathrm{Qt}$ & $\begin{array}{l}12110 \\
3013 \mathrm{~B} \\
\end{array}$ & $\begin{array}{c}\text { SAVY400 } \\
0 \\
\end{array}$ & Jan-00 & 0.0 & 4. $\mathrm{E}+07$ & 70 & NA \\
\hline & & 362 & $\begin{array}{c}\text { PLUTONIUM } \\
\text { SUSTAINME } \\
\text { NT-LANL } \\
\end{array}$ & HGO7240CP & 52 & Non-Std & NA & NA & $\begin{array}{l}\text { Non- } \\
\text { Standard }\end{array}$ & Nov-12 & 0.0 & 1.E+08 & 50 & NA \\
\hline \multirow[b]{2}{*}{$\mathrm{C} 80$} & \multirow{2}{*}{$\begin{array}{c}\text { COMPOUND, } \\
\text { Tetrafluoride }\end{array}$} & \multirow[b]{2}{*}{345} & \multirow{2}{*}{$\begin{array}{c}\text { MATLS } \\
\text { STAGING \& } \\
\text { DISPOSITIO } \\
\text { N- NON-NS }\end{array}$} & PH3F & 54 & Hagan & $3 \mathrm{Qt}$ & NA & SNMC-1 & Apr-05 & 7.6 & $2 . \mathrm{E}+07$ & 50 & 300 \\
\hline & & & & PH5R4 & 54 & Hagan & $3 \mathrm{Qt}$ & NA & SNMC-2 & Oct-06 & 6.1 & 7.E+06 & 25 & NA \\
\hline \multirow{3}{*}{$\mathrm{C} 21$} & \multirow{3}{*}{$\begin{array}{l}\text { COMPOUND, } \\
\text { Dioxide }\end{array}$} & 345 & $\begin{array}{c}\text { MATLS } \\
\text { STAGING \& } \\
\text { DISPOSITIO } \\
\text { N- NON-NS }\end{array}$ & RBS5657-1A & 56 & Hagan & $5 \mathrm{Qt}$ & NA & SNMC-2 & Apr-04 & 8.6 & $3 . \mathrm{E}+07$ & 45 & NA \\
\hline & & 362 & $\begin{array}{c}\text { PLUTONIUM } \\
\text { SUSTAINME } \\
\text { NT-LANL }\end{array}$ & CLO082911 & 52 & $\begin{array}{l}\text { SAVY- } \\
4000\end{array}$ & $5 \mathrm{Qt}$ & $\begin{array}{l}03110 \\
5052 \mathrm{~B}\end{array}$ & $\begin{array}{c}\text { SAVY400 } \\
0\end{array}$ & Jan-00 & 0.0 & 8.E+07 & 35 & 370 \\
\hline & & 650 & $\begin{array}{c}\text { MATERIAL } \\
\text { IDENTIFICA } \\
\text { TION AND } \\
\text { SURVEILLA } \\
\text { NCE } \\
\end{array}$ & PBO & 54 & $\begin{array}{l}\text { SAVY- } \\
4000\end{array}$ & $5 \mathrm{Qt}$ & $\begin{array}{l}03110 \\
5028 \mathrm{~B}\end{array}$ & $\begin{array}{c}\text { SAVY } 400 \\
0\end{array}$ & Jan-00 & 0.0 & 1.E+08 & 59 & 490 \\
\hline \multicolumn{15}{|c|}{ Second Choice Containers } \\
\hline \multirow[b]{2}{*}{ R83 } & \multirow[b]{2}{*}{$\begin{array}{l}\text { PROCESS } \\
\text { RESIDUE, } \\
\text { MSE Salt }\end{array}$} & \multirow[b]{2}{*}{716} & \multirow{2}{*}{$\begin{array}{c}\text { ISOTOPE } \\
\text { PRODUCTIO } \\
\text { N AND } \\
\text { DISTRIBUTI } \\
\text { ON } \\
\end{array}$} & CABL126B & 52 & Hagan & $5 \mathrm{Qt}$ & NA & SNMC-1 & Aug-05 & 7.3 & $6 . \mathrm{E}+07$ & 12 & 100 \\
\hline & & & & CABL129B & 52 & Hagan & $5 \mathrm{Qt}$ & NA & SNMC-1 & Aug-06 & 6.3 & 4. $\mathrm{E}+07$ & 35 & 150 \\
\hline $\mathrm{R} 78$ & $\begin{array}{c}\text { PROCESS } \\
\text { RESIDUE, } \\
\text { Sweepings/Scr } \\
\text { eenings }\end{array}$ & 322 & $\begin{array}{c}\text { SPECIAL } \\
\text { ACTINIDE } \\
\text { RECOVERY }\end{array}$ & YER242A & 42 & Hagan & $3 \mathrm{Qt}$ & NA & SNMC-1 & Aug-03 & 9.3 & 2. $\mathrm{E}+07$ & 7 & NA \\
\hline
\end{tabular}




\begin{tabular}{|c|c|c|c|c|c|c|c|c|c|c|c|c|c|c|}
\hline IDC & $\begin{array}{c}\text { IDC } \\
\text { Description }\end{array}$ & $\begin{array}{c}\text { Project } \\
\text { Code }\end{array}$ & $\begin{array}{c}\text { Project } \\
\text { Description }\end{array}$ & Item & DMT & $\begin{array}{l}\text { Initial } \\
\text { Containe } \\
\text { r Type }\end{array}$ & Size & $\begin{array}{c}\text { SAVY } \\
\text { SN }\end{array}$ & $\begin{array}{c}\text { Containe } \\
\mathbf{r} \\
\text { Category }\end{array}$ & $\begin{array}{l}\text { Hagan } \\
1^{\text {st }} \text { Use }\end{array}$ & $\begin{array}{l}\text { Hagan } \\
\text { Age } \\
\text { (yr) }\end{array}$ & $\begin{array}{l}\text { Calc } \\
\text { Dose }\end{array}$ & $\begin{array}{c}\text { B/G } \\
(\mathbf{m r e m} / \\
\mathbf{h})\end{array}$ & $\begin{array}{c}\mathbf{B} / \mathbf{G} \\
\text { w/o } \\
\text { shield } \\
\text { (mrem/ } \\
\text { h) }\end{array}$ \\
\hline R65 & $\begin{array}{c}\text { PROCESS } \\
\text { RESIDUE, ER } \\
\text { Salt }\end{array}$ & 345 & $\begin{array}{c}\text { MATLS } \\
\text { STAGING \& } \\
\text { DISPOSITIO } \\
\text { N- NON-NS }\end{array}$ & GBS015 & 52 & Hagan & $8 \mathrm{Qt}$ & NA & SNMC-2 & May-12 & 0.6 & 9. $\mathrm{E}+07$ & 40 & 300 \\
\hline \multirow{2}{*}{$\mathrm{R} 47$} & \multirow{2}{*}{$\begin{array}{c}\text { PROCESS } \\
\text { RESIDUE, } \\
\text { Incinerator Ash }\end{array}$} & \multirow{2}{*}{703} & \multirow{2}{*}{$\begin{array}{c}\text { DOMESTIC } \\
\text { PU238 } \\
\text { ACTIVITIES }\end{array}$} & TDC59 & 83 & Hagan & $8 \mathrm{Qt}$ & NA & SNMC-1 & Apr-01 & 11.6 & $9 . \mathrm{E}+07$ & 10 & NA \\
\hline & & & & TDC93 & 83 & Hagan & $8 \mathrm{Qt}$ & NA & SNMC-1 & Apr-01 & 11.6 & $9 . \mathrm{E}+07$ & 23 & 100 \\
\hline \multirow{6}{*}{$\mathrm{R} 26$} & \multirow{6}{*}{$\begin{array}{c}\text { PROCESS } \\
\text { RESIDUE, } \\
\text { Filter Residue }\end{array}$} & \multirow{3}{*}{345} & \multirow{3}{*}{$\begin{array}{c}\text { MATLS } \\
\text { STAGING \& } \\
\text { DISPOSITIO } \\
\text { N- NON-NS }\end{array}$} & FFU685R & 54 & Hagan & $5 \mathrm{Qt}$ & NA & SNMC-2 & Jun-05 & 7.5 & 2.E+07 & 3 & NA \\
\hline & & & & FFU786R & 54 & Hagan & $3 \mathrm{Qt}$ & NA & SNMC-1 & Jun-07 & 5.5 & 2.E+07 & 25 & 700 \\
\hline & & & & VTB10C1 & 52 & Hagan & $5 \mathrm{Qt}$ & NA & SNMC-1 & Aug-05 & 7.3 & $5 . \mathrm{E}+07$ & 30 & 250 \\
\hline & & \multirow{2}{*}{362} & \multirow{2}{*}{$\begin{array}{c}\text { PLUTONIUM } \\
\text { SUSTAINME } \\
\text { NT-LANL } \\
\end{array}$} & RESED82A & 52 & Hagan & $3 \mathrm{Qt}$ & NA & SNMC-3 & Oct-01 & 11.1 & 2.E+07 & 35 & $\mathrm{NA}$ \\
\hline & & & & RESED86B & 52 & Hagan & $3 \mathrm{Qt}$ & NA & SNMC-2 & Aug-03 & 9.3 & $2 . \mathrm{E}+07$ & 35 & 90 \\
\hline & & 419 & ARIES & ATLBL6/5-1 & 52 & Hagan & $5 \mathrm{Qt}$ & $\mathrm{NA}$ & SNMC-2 & Aug-05 & 7.3 & 4.E+07 & 60 & NA \\
\hline \multirow{3}{*}{ N50 } & \multirow{3}{*}{$\begin{array}{c}\text { NON- } \\
\text { COMBUSTIB } \\
\text { LE, MgO }\end{array}$} & \multirow{3}{*}{345} & \multirow{3}{*}{$\begin{array}{c}\text { MATLS } \\
\text { STAGING \& } \\
\text { DISPOSITIO } \\
\text { N- NON-NS }\end{array}$} & BLC9343 & 56 & Hagan & $3 \mathrm{Qt}$ & $\mathrm{NA}$ & SNMC-1 & Jul-08 & 4.4 & $1 . \mathrm{E}+07$ & 10 & 240 \\
\hline & & & & BRC37 & 52 & Hagan & $12 \mathrm{Qt}$ & NA & SNMC-2 & Oct-05 & 7.1 & $6 . \mathrm{E}+06$ & 12 & 220 \\
\hline & & & & MGCA0102 & 52 & Hagan & $3 \mathrm{Qt}$ & NA & SNMC-3 & Aug-06 & 6.3 & $5 . \mathrm{E}+06$ & 9 & 100 \\
\hline $\mathrm{C} 80$ & $\begin{array}{c}\text { COMPOUND, } \\
\text { Tetrafluoride }\end{array}$ & 345 & $\begin{array}{c}\text { MATLS } \\
\text { STAGING \& } \\
\text { DISPOSITIO } \\
\text { N- NON-NS }\end{array}$ & PMT1-003 & 54 & Hagan & $5 \mathrm{Qt}$ & NA & SNMC-2 & Jan-09 & 3.9 & 9. $\mathrm{E}+06$ & 22 & NA \\
\hline $\mathrm{C} 21$ & $\begin{array}{c}\text { COMPOUND, } \\
\text { Dioxide }\end{array}$ & 345 & $\begin{array}{c}\text { MATLS } \\
\text { STAGING \& } \\
\text { DISPOSITIO } \\
\text { N- NON-NS } \\
\end{array}$ & RBS1-A & 54 & Hagan & $5 \mathrm{Qt}$ & NA & SNMC-1 & Nov-02 & 10.0 & 2. $\mathrm{E}+08$ & 82 & 180 \\
\hline
\end{tabular}


Table 2. Target schedule for the twenty-four containers with worst case materials chosen for field shelf-life surveillance program.

\begin{tabular}{|c|c|c|c|c|}
\hline IDC & ITEM & $\begin{array}{c}\text { Target Packaging } \\
\text { Date (quarter / cal. } \\
\text { year) }\end{array}$ & $\begin{array}{c}\text { Target Surveillance } \\
\text { Date }(\geq \text { quarter / cal. } \\
\text { year })\end{array}$ & $\begin{array}{c}\text { Age at } \\
\text { Surveillance }\end{array}$ \\
\hline $\mathrm{N} 50$ & BLC9413 & $1 \mathrm{st} / 2013$ & $1 \mathrm{st} / 2014$ & One year \\
\hline M74 & 63NLBE & 1 st / 2013 & 1st/ 2014 & One year \\
\hline $\mathrm{C} 80$ & PH3F & $1 \mathrm{st} / 2013$ & $1 \mathrm{st} / 2014$ & One year \\
\hline $\mathrm{C} 21$ & $\begin{array}{l}\text { RBS5657-1A } \\
\end{array}$ & 1 st / 2013 & 1st/2014 & One year \\
\hline $\mathrm{R} 26$ & TKS1C1 (SAVY) & 1 st / 2012 & $1 \mathrm{st} / 2014$ & Two years \\
\hline N50 & ORF633956BLC & 1 st / 2013 & 1 st / 2015 & Two years \\
\hline M44 & HGO7240CP (non-std) & 1 st / 2013 & $1 \mathrm{st} / 2015$ & Two years \\
\hline $\mathrm{C} 80$ & PH5R4 & 1 st / 2013 & 1 st / 2015 & Two years \\
\hline R71 & 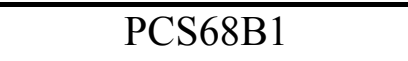 & 1 st / 2013 & $1 \mathrm{st} / 2016$ & Three years \\
\hline R65 & $\overline{\text { GBS005 }}$ & 1 st / 2013 & 1 st / 2016 & Three years \\
\hline $\mathrm{R} 47$ & INCA-21 & $1 \mathrm{st} / 2013$ & $1 \mathrm{st} / 2016$ & Three years \\
\hline $\mathrm{R} 42$ & SLTF3123A & $1 \mathrm{st} / 2013$ & $1 \mathrm{st} / 2016$ & Three years \\
\hline $\mathrm{C} 21$ & PBO (SAVY) & $1 \mathrm{st} / 2012$ & $1 \mathrm{st} / 2016$ & Four years \\
\hline $\mathrm{R} 83$ & BLS25 & $2 \mathrm{nd} / 2013$ & 2nd / 2017 & Four years \\
\hline R78 & $\mathrm{PO} 4275 \mathrm{C} 1$ & 2nd / 2013 & 2nd / 2017 & Four years \\
\hline $\mathrm{R} 47$ & INCA-20 & 2nd / 2013 & $2 \mathrm{nd} / 2017$ & Four years \\
\hline R78 & VTB-16C1 & 2nd / 2013 & 2nd / 2018 & Five years \\
\hline R71 & SWPVTB15 & 2nd / 2013 & 2nd / 2018 & Five years \\
\hline $\mathrm{R} 65$ & GBS059 & 2nd / 2013 & 2nd / 2018 & Five years \\
\hline $\mathrm{R} 42$ & BSO153 & 2nd / 2013 & 2nd / 2018 & Five years \\
\hline $\mathrm{R} 83$ & CABL128D & $1 \mathrm{st} / 2013$ & $\begin{array}{c}\text { Annual NDE / DE in } \\
2016 \text { or later }\end{array}$ & $\geq$ Four Years \\
\hline $\mathrm{R} 26$ & ROTRB9C2 (SAVY) & $3 \mathrm{rd} / 2011$ & $\begin{array}{c}\text { Annual NDE / DE in } \\
2016 \text { or later }\end{array}$ & $\geq$ Four Years \\
\hline M44 & NAB183-2 (SAVY) & 2nd / 2012 & \begin{tabular}{|c|} 
Annual NDE / DE in \\
2016 or later \\
\end{tabular} & $\geq$ Four Years \\
\hline $\mathrm{C} 21$ & CLO082911 (SAVY) & $1 \mathrm{st} / 2012$ & $\begin{array}{c}\text { Annual NDE / DE in } \\
2016 \text { or later }\end{array}$ & $\geq$ Four Years \\
\hline
\end{tabular}




\section{SURVEILLANCE MEASUREMENTS}

The main focus of both field shelf-life surveillance and items of opportunity surveillance is to evaluate O-rings, filters and the container's condition, particularly for any sign of performance degradation, corrosion or leakage. Since the container has a filter, pressurization could be an indication of a plugged filter and could increase the potential for loss of containment. Measurements and examinations to assess potential problems will be conducted on all surveillance containers. Some of these evaluations provide "yes/no" data and others provide quantitative data. In the descriptions below, the quantitative data are shown in bold.

\section{Non-Destructive Evaluations (NDE) for SAVY-4000 Containers}

- Evaluations during the unpacking of the outer container:

- Any visual indications of pressurization (slight bulging) or corrosion? (yes/no)

- Does visual inspection show signs of any dents or gouges that may have occurred in normal handling or possibly due to dropping the container? (yes/no)

- Weight measurement (trends will be evaluated to see if there is an indication of moisture absorption or metal oxidation).

- Do contamination surveys show any indication of O-ring seal failure, weld failure or filter failure? (yes/no)

- Visual examination to determine inner package condition and if the container was package according to procedures:

$\circ$ O-ring installed? (yes/no)

- Inner container consistent with material form, e.g., stainless steel slip lid for oxide, hermetically sealed for metal, etc.? (yes/no)

○ Bag-out bag present? (yes/no)

- Bag-out bag intact, e.g., contamination found outside bag out bag indicates breached bag? (yes/no)

- Any liquid observed inside the bag-out bag? (yes/no)

- Metal inner container intact (e.g., no holes corroding through, no plutonium in contact with bag out bag, etc.) (yes/no)

- Evaluations on the empty SAVY-4000 container:

Do the interior and/or exterior surfaces of the container show any signs of corrosion or discoloration?

- Does the functional check of the closure system show any impingement of moving parts impeding closure? (yes/no)

- Does the O-ring groove on the body collar show any signs of damage, i.e., scratches, burr, etc.? (yes/no)

- Does additional non-destructive testing of containment barrier welds (as deemed necessary by a subject matter expert) show any positive indication of weld cracking or other weld flaws? (yes/no)

○ O-Ring: 
- Does the SAVY 4000 O-ring need replacement based on the visual inspection: scratches, cuts or other damage on the o-ring itself that might prevent an effective seal? (yes/no)

- Leakage testing shall be in accordance with ANSI N14.5. The test pass rate shall be equal to the design release rate established as $5.6 \times 10^{-6} \mathrm{~cm}^{3} \mathrm{~s}^{-1}$ of fluid. The testing helium leak rate criterion is $1.0 \times 10^{-5} \mathrm{~atm} \mathrm{~cm}^{3} \mathrm{~s}^{-1}$ at a differential pressure of $10 \mathrm{kPa}$.

- The Shore M hardness will be measured and evaluated using Parker o-ring manufacturer recommended tolerances.

- Filter Function:

- Based on visual inspection of the outside of the lid, does the area around the filter show indications of particulate escape? (Particulates could indicate that the filter is compromised.) (yes/no)

- Based on visual inspection of the inside and the outside of the lid, does the filter material itself show indications of discoloration or occlusion? (yes $/$ no)

- Aerosol filter test at a flow rate of $\sim 200 \mathrm{ml} / \mathrm{min}$

- $\%$ penetration $(<0.03 \%)$

- Pressure drop across the filter $(<1$ inch water column)

- Does the filter pass the water penetration test (yes/no, at 12 inches water column pressure on the outside of the lid, pass=no visual indication of water penetration on the inside of the lid for at least 60 seconds)?

\section{Destructive Evaluations (DE)}

In addition to the NDE measurements and evaluations, which will be performed on all surveillance containers as noted above, detailed (destructive) evaluations of O-rings will be performed on the field surveillance sample and possibly on items-of-opportunity requiring replacement of the O-ring. These data will be used to supplement ongoing accelerated aging studies (Blair, et. al., 2012) In the same manner as the accelerated aging studies, destructive evaluation (DE) tests for the SAVY and Hagan O-rings will consist of mechanical testing, thermal properties testing, and spectroscopic measurements. The mechanical testing measurements will comprise Shore $\mathrm{M}$ hardness, compression stress relaxometry, compression set, and tensile testing. The thermal testing will consist of either Differential Scanning Calorimetry (DSC) or Thermogravimetric Analysis (TGA). Fourier Transform Infrared (FTIR) spectroscopic measurements will be used along other techniques that provide chemical characterization of material changes. Combining these three approaches permits a more complete evaluation of the aging characteristics of the O-rings. ${ }^{1}$

\section{SURVEILLANCE DATABASE}

A key component of the surveillance program is the surveillance database. LANL has initiated the development of a database with relevant information about stored materials and containers, including material, packaging, and surveillance information for each storage container. The database will document important information for trending studies such as the age of the container, how long it has

\footnotetext{
${ }^{1}$ Hagan container O-rings will also be examined using these same approaches on a funds-available basis.
} 
been in service, its in-service history (e.g., what materials have been loaded for what periods of time), and the dates of replacements of key components. The database will provide such information as well as information about the materials in these containers, so that a material causing potential problems to the inner container can be identified. The database will also be used to document the inspection/testing results from this plan and from the lifetime extension studies.

\section{REFERENCES}

DOE M 441.1-1, Nuclear Material Packaging Manual, U.S. Department of Energy, 2008.

Anderson, Luke L., Blair, Michael W., Hamilton, E. Jeanne, Kelly, Elizabeth J., Moore, Murray E., Smith, Paul H., Stone, Timothy A., Teague, Jonathan G., Veirs, D. Kirk, Weis, Eric and Yarbro, Tresa F., 2012, Safety Analysis Report for the SAVY 4000 Container Series, Revision 1, LA-CP12-00204.

Blair, M.W., Weis, E. M., Veirs, D. K., Stone, T. M., Smith, P. H. 2012. Accelerated aging studies for the lifetime extension of O-rings used in the SAVY-400 unit. Los Alamos National laboratory Report, LA-UR-12-00747.

Smith, P.H., Jordan, H., Hoffman, J.A., Eller, P.G., and Balkey, S., May, 2007, Risk Ranking of LANL Nuclear Material Storage Containers for Repackaging Prioritization, Operational Radiation Safety, Vol. 92, suppl 2, p. S87-S97. 


\section{Appendix A. Detecting an important trend over time with high confidence}

Although the four items evaluated each year to determine if there is an aging trend are worst case items not a random sample, these items will provide results that will be biased in a conservative direction. Therefore the results are bounding as compared to a random sample.

If a random sample were used, at the end of four years there would be a $95 \%$ probability (the power) of detecting a trend that is at least one and half times the standard deviation $(\sigma)$ of the measurements. At the end of five years, the power would be over $99.9 \%$ for a trend at least one and half times $\sigma$ and the power would be $95 \%$ for a trend at least 0.75 times $\sigma$. These calculations are based on a Type I error of $10 \%$ (e.g., a $10 \%$ chance of saying there is a trend when there is no trend).

This calculation is based on results given in the paper "Designing Monitoring Programmes for Detecting Temporal Trends in Contaminants in Fish and Shellfish," by M.D. Nicholson, R.J. Fryer and C. A. Ross, Marine Pollution Bulletin, Vol. 34, No. 10, pp 821-826, 1997. Using the notation in the paper, the average of the measurements for a given year for a given measurement is denoted $\mathrm{y}_{\mathrm{t}}$, where $\mathrm{t}$ indees over time, e.g., $\mathrm{t}=1,2, \ldots 5$, and

$\mathrm{E}\left(\mathrm{y}_{\mathrm{t}}\right)=a+b \mathrm{t}$ and $\operatorname{Var}\left(\mathrm{y}_{\mathrm{t}}\right)=\psi^{2}=\sigma^{2} / 4$ (in our application).

Evidence of a linear trend is determined by regression of $\mathrm{y}_{\mathrm{t}}$ on $t$ and testing the null hypothesis that $b=$ 0 . If the Type I error $=10 \%$, then the power after $\mathrm{T}$ years (e.g., 4 ) is given by a non-central $\mathrm{F}$ distribution with non-centrality parameter $\delta$,

power $=1-\operatorname{PROB}\left[\mathrm{F}\left(q f_{0.90}, 1,3, \delta\right)\right]$, where

$q f_{0.90}$ is the quantile of the central $\mathrm{F}, \mathrm{F}(0.9,1,3)$ and

$\delta=b^{2} \frac{(T-1) T(T+1)}{12 \psi^{2}}$.

Applying these equations for a ratio of the trend $(b)$ to $\sigma$ (standard deviation of the measurements) of 1.5 and using the non-central $\mathrm{F}$ calculator found at $\underline{h t t p}$ ://www.danielsoper.com/statcalc/calc06.asp , the power of detecting such a trend after four years is found to be slightly larger than $95 \%$.

In addition to these DE trend data, NDE trend data for the four items that are evaluated over time will be available. Evaluating the same items over time provides better information for trend analysis, since trends for each item can be evaluated. This is particularly useful in cases where some items are trending upwards, others downward or not at all. The results of this item-by-item trend analysis will support interpretation of the results from the other trend analyses. 


\section{Appendix B. Traveler for Repackaging Items into SAVY-4000 Containers}

\section{Background:}

The purpose of this traveler is to capture some essential information during or shortly after the Hagan containers (and one other non-standard container) are repackaged into SAVY-4000 containers. This information is required to develop a good understanding of the effects of long-term storage and radiation dose to the containers, particularly the Hagan o-rings.

\section{General Instructions:}

If possible, the items should be repackaged into the same size SAVY-4000 container that they are currently packaged in. For those items that are currently in Hagan containers, the Hagan container, once it is determined to be free of contamination, should be saved for testing. Please call Judy Sanchez or Paul Smith to have them pick up the container and this traveler. If the bag-out bag on the inner container is intact (not brittle, not sticking to inner container, no contamination on outside) the item can be placed directly into the new SAVY-4000 container. If the item must be introduced into the glove-box, remove the old bag-out bag, replace the inner container if necessary (i.e., if it is corroded, non-metal, etc.), and then bag-out the item. If shielding is necessary to reduce the external dose on the new SAVY-4000, please use external pewter shielding only (assuming that is sufficient). If there is lead or other types of shielding inside the old container, please do not transfer it to the new SAVY4000 container. 
Appendix B (continued). Traveler for Repackaging Items into SAVY-4000 Containers

Information requested for the old container (Mostly Hagans, one non-standard):

Date of repackaging:

Person performing the repackaging: $\mathrm{Z \#}$ Last Name First Name

Hagan Filter Serial \#:

Hagan Body Serial \#: Item (LOTID): LAMCAS Transaction ID:

Internal shielding present (circle): Yes No

Type of inner shielding (circle): Lead Pewter Other (please specify)

Condition of old outer container (circle): corrosion dented outer contamination Condition of old bag-out bag (circle): brittle stuck to inner container outer contamination other (please specify)

Beta/Gamma Dose (at the lid/collar position on the Hagan):

Beta/Gamma Dose (at $30 \mathrm{~cm})$ :

Neutron Dose:

Comments:

Information requested for the new container (SAVY-4000):

Serial \# on the SAVY-4000 lid:

Serial \# on the SAVY-4000 body:

Item (LOTID): LAMCAS Transaction ID:

Type of inner container (circle): stainless steel slip lid hermetically sealed (type):

Pewter shielding present (circle): Yes No

Type of pewter shielding used (circle): Internal External

As-Packaged Weight of SAVY-4000:

Beta/Gamma Dose just above the filter:

Beta/Gamma Dose at the container collar:

Neutron Dose:

Comments: 


\section{Appendix C. Table of Acronyms}

\begin{tabular}{|c|c|}
\hline Acronym & Definition \\
\hline $\mathrm{IDC}^{*}$ & Item Description Code \\
\hline SMT & Summary Material Type \\
\hline DMT & Detail Material Type \\
\hline $\mathrm{DOE}$ & Department of Energy \\
\hline LANL & Los Alamos National Laboratory \\
\hline $\mathrm{DE}$ & Destructive Examination \\
\hline CEDE & Committed Effective Dose Equivalent \\
\hline ID & Identification \\
\hline SN & Serial Number \\
\hline $\mathrm{B} / \mathrm{G}$ & Beta/Gamma \\
\hline SNMC & Standard Nuclear Material Container \\
\hline SAVY -4000 & $\begin{array}{l}\text { Nuclear Material Storage Container } \\
\text { Designed for M441.1-1 Compliance }\end{array}$ \\
\hline MSE & Molten Salt Extraction \\
\hline NS & National Security \\
\hline ER & Electrorefining \\
\hline DOR & Direct Oxide Reduction \\
\hline NDE & Non-Destructive Examination \\
\hline
\end{tabular}

*The IDC codes used in this plan are abbreviated to include the $1^{\text {st }}$ three characters. 
E. Kelly, P. Smith, D. K. Veirs, T. Stone, D. Prochnow, L. Worl, E. Weis, M. Blair

\section{Appendix D. Table of Item Description Code (IDC) Definitions}

\begin{tabular}{|c|c|}
\hline IDC $^{*}$ & DESCRIPTION \\
\hline $\mathrm{C} 13$ & COMPOUND; Carbide \\
\hline $\mathrm{C} 19$ & COMPOUND; Chloride \\
\hline $\mathrm{C} 21$ & COMPOUND; Dioxide \\
\hline $\mathrm{C} 28$ & COMPOUND; Fluoride \\
\hline $\mathrm{C} 40$ & COMPOUND; Hydride \\
\hline $\mathrm{C} 52$ & COMPOUND; Nitrate \\
\hline $\mathrm{C} 54$ & COMPOUND; Nitride \\
\hline $\mathrm{C} 77$ & COMPOUND; Sulfate \\
\hline $\mathrm{C} 80$ & COMPOUND; Tetrafluoride \\
\hline $\mathrm{C} 82$ & COMPOUND; Trichloride \\
\hline $\mathrm{C} 86$ & COMPOUND; Trioxide \\
\hline $\mathrm{C} 88$ & COMPOUND; U3O8 \\
\hline E13 & REACTOR ELEMENT; Carbide \\
\hline E88 & REACTOR ELEMENT; U3O8 \\
\hline K00 & COMBUSTIBLE; Non Specific \\
\hline K60 & COMBUSTIBLE; Paper/Wood \\
\hline M32 & METAL; Beryllide \\
\hline M44 & METAL; Unalloyed Metal \\
\hline M74 & METAL; Alloyed Metal \\
\hline N00 & NON-COMBUSTIBLE; Non Specific \\
\hline $\mathrm{N} 24$ & NON-COMBUSTIBLE; Filter Media \\
\hline N29 & NON-COMBUSTIBLE; Glass \\
\hline N31 & NON-COMBUSTIBLE; Graphite \\
\hline N35 & NON-COMBUSTIBLE; HEPA Filter(s) \\
\hline N50 & NON-COMBUSTIBLE; MgO \\
\hline N55 & NON-COMBUSTIBLE; Non-Actinide Metal \\
\hline N67 & NON-COMBUSTIBLE; Plastics/Kim Wipes \\
\hline N69 & NON-COMBUSTIBLE; Resin \\
\hline R03 & PROCESS RESIDUE; Hydrogenous Salt \\
\hline R09 & PROCESS RESIDUE; Calcium Salt \\
\hline $\mathrm{R} 12$ & PROCESS RESIDUE; Calcium Metal \\
\hline $\mathrm{R} 26$ & PROCESS RESIDUE; Filter Residue \\
\hline R41 & PROCESS RESIDUE; Hydroxide Precipitate \\
\hline R42 & PROCESS RESIDUE; DOR Salt \\
\hline R47 & PROCESS RESIDUE; Incinerator Ash \\
\hline R59 & PROCESS RESIDUE; Oxalate Precipitate \\
\hline R65 & PROCESS RESIDUE; ER Salt \\
\hline R71 & PROCESS RESIDUE; Salt \\
\hline R73 & PROCESS RESIDUE; Silica \\
\hline R78 & PROCESS RESIDUE; Sweepings/Screenings \\
\hline R83 & PROCESS RESIDUE; MSE Salt \\
\hline
\end{tabular}




\section{Appendix E. Table of Summary Material Type (SMT) Definitions}

\begin{tabular}{|c|c|}
\hline SMT & DESCRIPTION \\
\hline 10 & Uranium - Depleted in U-235 \\
\hline 20 & Uranium - Enriched in U-235 \\
\hline 40 & Plutonium - Pu-242 \\
\hline 44 & Americium - Am-241 \\
\hline 45 & Americium - Am-243 \\
\hline 46 & Curium \\
\hline 50 & Plutonium - Pu-239 \\
\hline 70 & Uranium - Enriched in U-233 \\
\hline 81 & Uranium - Normal $(0.7117 \%$ U-235) \\
\hline 82 & Neptunium - Np-237 \\
\hline 83 & Plutonium - Pu-238 \\
\hline 86 & Deuterium - D-2 \\
\hline 88 & Thorium \\
\hline
\end{tabular}

